\title{
Anticipatory Postural Adjustments During Lateral Step Motion in Patients With Hip Osteoarthritis
}

\section{$\operatorname{AUTHOR(S):~}$}

Tateuchi, Hiroshige; Ichihashi, Noriaki; Shinya, Masahiro; Oda, Shingo

\section{CITATION:}

Tateuchi, Hiroshige ... [et al]. Anticipatory Postural Adjustments During Lateral Step Motion in Patients With Hip Osteoarthritis. Journal of Applied Biomechanics 2011, 27(1): 32-39

ISSUE DATE:

2011-02

URL:

http://hdl.handle.net/2433/141843

RIGHT:

(c) 2011 Human Kinetics, Inc. 


\title{
Anticipatory Postural Adjustments During Lateral Step Motion in Patients With Hip Osteoarthritis
}

\author{
Hiroshige Tateuchi, Noriaki Ichihashi, Masahiro Shinya, and Shingo Oda
}

\begin{abstract}
Patients with hip osteoarthritis (OA) have difficulty with mediolateral postural control. Since the symptom of hip OA includes joint pain, which mostly occurs upon initial movement, patients with hip OA might have disabling problems with movement initiation. This study aimed to identify the movement strategy during the anticipatory postural adjustments in the lateral step motion in patients with hip OA. We studied 18 female subjects with unilateral hip OA and 10 healthy subjects, and measured temporal, kinetic, and kinematic variables. Patients with hip OA required a longer duration of anticipation phase than the control subjects, the total duration of lateral stepping was not different between the groups. Displacement of the center of mass to the supporting (affected) side during the anticipation phase was not different between the two groups. These findings suggest that, in patients with hip OA, the center of mass slowly moved to the affected side. Furthermore, patients with hip OA showed greater shift of the trunk to the supporting side than did the control subjects. These movement characteristics might contribute to the achievement of both protection of the affected hip joint and quickness in the subsequent lateral step in patients with hip OA.
\end{abstract}

Keywords: postural control, hip joint, movement initiation

Osteoarthritis of the hip (hip OA) is the most common cause of musculoskeletal disability in developed countries, and the prevalence of hip OA in the general adult population is approximately 8\% (Dagenais et al., 2009; Quintana et al., 2008). It is higher in women than in men and tends to increase with age (Quintana et al., 2008). The clinical symptoms of hip OA include joint pain and stiffness, occurring mainly on joint motion and weight bearing, and progressive loss of joint function (Brown, 2006). Mediolateral stability is thought to be controlled mainly by the hip joint (Rogers \& Mille, 2003; Rogers \& Pai, 1993). Therefore, patients with hip OA might have potentially significant problems in performing motor tasks that involve mediolateral postural control such as a lateral stepping. There is in fact some evidence of individuals with hip OA falling frequently compared with age-matched healthy individuals (Arnold \& Faulkner, 2007). Thus, it is important to investigate mediolateral postural control in patients with hip OA in a lateral stepping task.

Hiroshige Tateuchi (Corresponding Author) is with the Graduate School of Medicine and the Graduate School of Human and Environmental Studies, Kyoto University, Kyoto, Japan. Noriaki Ichihashi is with the Graduate School of Medicine, Kyoto University, Kyoto, Japan. Masahiro Shinya is with the Graduate School of Human and Environmental Studies, Kyoto University, Kyoto, Japan. Shingo Oda is with the Graduate School of Human and Environmental Studies, Kyoto University, Kyoto, Japan.
Lateral stepping is a frequently repeated action in our daily life, and it plays an important role in postural control, for example, change-in-support strategies (Maki \& McIlroy, 1997). A disability in mediolateral postural control is associated with future risk of falls (Maki et al., 1994), and its failure directly leads to falls and serious injuries such as hip fractures (Rogers \& Mille, 2003). Lateral stepping is a complex motor task that entails the transition from a quiet standing posture with double-limb support to dynamic equilibrium that allows body progression. The movement of the stepping leg is preceded by postural adjustments serving to shift the center of mass (CoM) toward the supporting side so that the leg can be raised (Brenière et al., 1987). These preparatory controls are called "anticipatory postural adjustments" (Lepers \& Breniére, 1995; Shumway-Cook \& Woollacott, 2001). Shifting the CoM toward the supporting side during anticipation phase before initiating a lateral step shortens the distance between the CoM of the trunk segment and the center of rotation of the supporting hip joint and decreases the gravity torque of the trunk on the supporting hip joint, which reduces stress on the supporting limb during the single-stance phase of the subsequent lateral stepping. At the same time, however, shifting the CoM toward the supporting side is accomplished by loading the supporting limb, which itself is a stress on the supporting limb during the anticipation phase. Further, shifting the CoM toward the supporting side would be disadvantageous for rapid lateral step motion because it moves diametrically opposite to the stepping direction. How do the patients with hip OA resolve this dilemma? It 
is possible that patients with hip OA resolve this problem by changing their movement strategy to meet the requirements to reduce the force acting on the affected hip joint and to step rapidly.

Prolonged anticipation phase accompanied by a large displacement of the acromion toward the supporting side before initiating the subsequent lateral step has been reported in patients with knee OA (Viton et al., 2002). Given the fact that the lateral weight transfer during the anticipation phase before lateral stepping is mainly controlled by the torque generated in the hip joint (Rogers \& Pai, 1993) and the joint pain of the hip OA is usually most pronounced upon initial movement (Bellamy et al., 1999), the postural control during anticipation phase in lateral stepping could be more difficult for patients with hip OA. It was demonstrated that the activity of the gluteus medius on the support side was greater during lateral step-up than during forward step-up (Mercer et al., 2009). This finding suggests that a step in the lateral direction is more challenging than a forward step because a lateral step involves greater activity of the hip muscles. However, no study has been conducted on the postural control of patients with hip OA in the lateral stepping task.

The aim of this study, therefore, was to identify the movement strategy during the anticipation phase in the lateral step motion in patients with hip OA. We hypothesized that patients with hip OA would display prolonged anticipation phase compared with healthy individuals. Prolonged anticipation phase would contribute to slow motion of the CoM and consequently decreases the stress on the affected hip joint. Further, patients with hip OA shift or incline the trunk and pelvis toward the supporting side when standing on one leg or during walking to shorten the distance between the CoM of the trunk segment and the center of rotation of the supporting hip joint and decrease the gravity torque of the trunk, which reduces stress on the supporting hip joint (Hardcastle \& Nade, 1985; Murray et al., 1971; Thurston, 1985; Watelain et al., 2001). On the basis of the previous kinematic data, we also hypothesized those patients with hip OA would shift or tilt their trunk which would be accompanied by shift of the CoM in the anticipation phase to lower the force acting on the affected hip joint.

\section{Methods}

Eighteen female subjects [age, $59.2 \pm 6.2$ years; weight, $51.8 \pm 7.1 \mathrm{~kg}$; height, $150.2 \pm 5.1 \mathrm{~cm}$; values are represented as the mean $\pm S D$ ] with unilateral hip OA (the OA group) scheduled for total hip arthroplasty volunteered to participate in this study. The exclusion criteria were symptomatic arthritis or deformities in any other lower extremity/spinal joints, more than a 3-cm discrepancy in the limb length, and neurological pathologies that could influence stepping motion. The mean score for the pain visual analog scale $(0-100 \mathrm{~mm})$ during weight bearing on the affected side while standing was $17.1 \pm 17.8 \mathrm{~mm}$.
On radiographic assessment, the mean sharp angle was found to be $44.3 \pm 4.3^{\circ}$ on the affected side. The sharp angle is commonly used to assess the degree of acetabular dysplasia (normal angle, 33-38 ${ }^{\circ}$ (Sharp \& England, 1961; Nagasawa et al., 2000). This study also included a control group of 10 healthy subjects matched for age, gender, weight, and height (age, 60.6 \pm 3.7 years; weight, $55.0 \pm 9.3 \mathrm{~kg}$; height, $152.1 \pm 6.3 \mathrm{~cm}$ ). The exclusion criteria for the control group were arthritis, injury to any joint in the lower extremity/spinal joints, a history of lower extremity/spinal surgery, and neurological disease. This study was carried out in accordance with the World Medical Association Declaration of Helsinki, and written informed consent was obtained from all the subjects before their participation in the study.

Each subject was instructed to stand barefoot on a dual-force platform system (Advanced Mechanical Technology Inc., Watertown, MA, USA) with one foot on one force platform, with the medial malleoli $10-\mathrm{cm}$ apart and the hands folded behind the back (Figure 1a). Movement during each trial started after the presentation of a visual cue using two (right and left) light-emitting diodes set anteroinferior to the subjects. The illumination of one of the diodes indicated to the subject which leg to step in the lateral direction. To avoid a preparatory weight shift, the subjects were directed to distribute their weight on both their legs as evenly as possible in the initial position, and the order in which the diodes illuminated was randomized. The subjects were instructed to step laterally covering a distance of 10 to $20 \mathrm{~cm}$ from the fifth metatarsal head of the stepping foot in the initial position. The subjects were instructed to perform the task as rapidly as possible in response to the visual cue and to maintain the final position for at least $3 \mathrm{~s}$ (Figure 1b). Sufficient practice was allowed before testing to familiarize the subjects with the required movements. Measurement was rejected when more than half of the foot failed to step within the setting range. For the OA group, steps with the affected limb supported were included in the analysis; for the control group, steps with the nondominant leg supported (i.e., the leg opposite to the one the subjects would use for kicking a ball) were included.

The ground reaction force was measured using force platforms at a sampling frequency of $1,080 \mathrm{~Hz}$. Kinematic measurements were performed using VICON 460 (Oxford Metrics Ltd., Oxford, UK) with six cameras operating at a sampling frequency of $120 \mathrm{~Hz}$. Six lightreflecting markers were attached to anatomical landmarks on both sides: the acromion, anterior-superior iliac spine, and greater trochanter (Figure 1).

The ground reaction forces in the frontal plane recorded from each of the force platforms were quantified. The peak mediolateral ground reaction force which was defined as the force that moves the CoM to the supporting side (Fx-support) and to the stepping side (Fx-step) during the anticipation phase was measured. In step initiation, the anticipation phase has been defined as the period from the onset of the center of pressure (CoP) shift to the foot-off of the stepping leg (Gélat et 
al., 2006; Liu et al., 2003). The Fx-support and Fx-step were standardized by the body weight of each subject.

The CoP was calculated from the resultant vertical ground reaction force recorded from the force platforms. The onset of CoP shift was determined to have occurred when the amplitude exceeded $3 S D$ from the mean amplitude of the baseline period $(1,500-\mathrm{ms}$ period before the onset of the visual cue: initial CoP position) for more than $50 \mathrm{~ms}$. The foot-off can be identified from the kinetic recording and was defined as the decrease in loading on the force plate under the stepping leg to $<10 \mathrm{~N}$. The footon was defined as the increase in loading on the force plate to the same extent $(10 \mathrm{~N})$. The peak displacement of the CoP shift toward the stepping side (CoP-step) and that toward the supporting side (CoP-support) during the anticipation phase were calculated from the initial $\mathrm{CoP}$ position (Figure 2).

The displacement of the CoM in the frontal plane was calculated from the resultant horizontal ground reaction force. The displacement of CoM can be obtained by using double integration of the acceleration. This technique has a disadvantage in that very small errors in force recordings are amplified by the double integration; we adopted the method developed by Lyon \& Day (1997) to correct
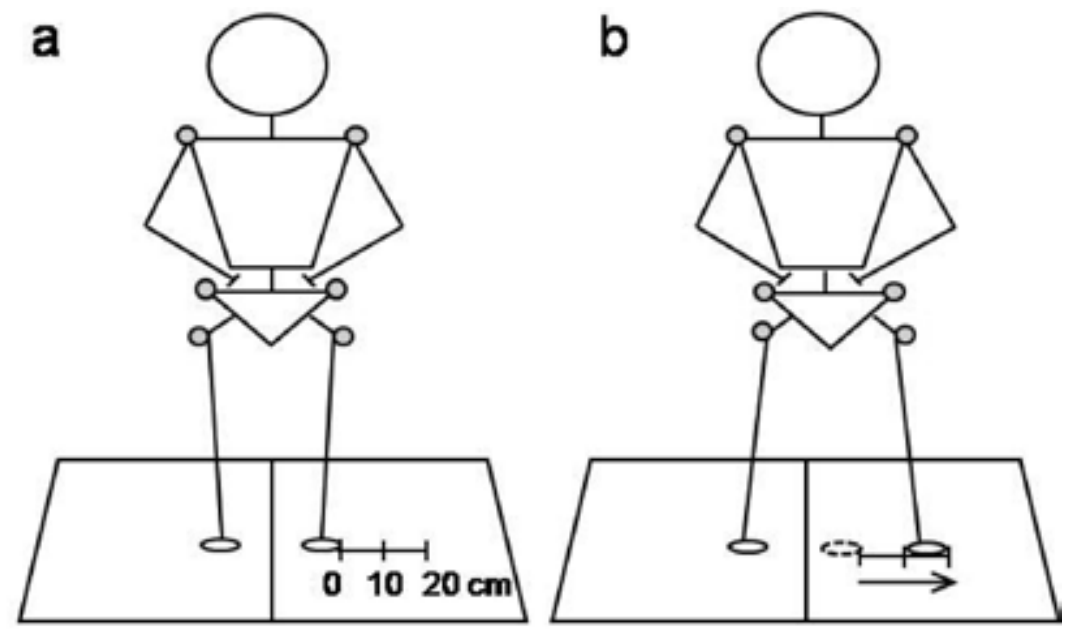

\section{Dual-force platform system}

Figure 1 - Initial position (a) and final position (b) of the lateral step motion. Six light-reflecting markers were bilaterally attached to anatomical landmarks: the acromion, anterior-superior iliac spine, and greater trochanter.

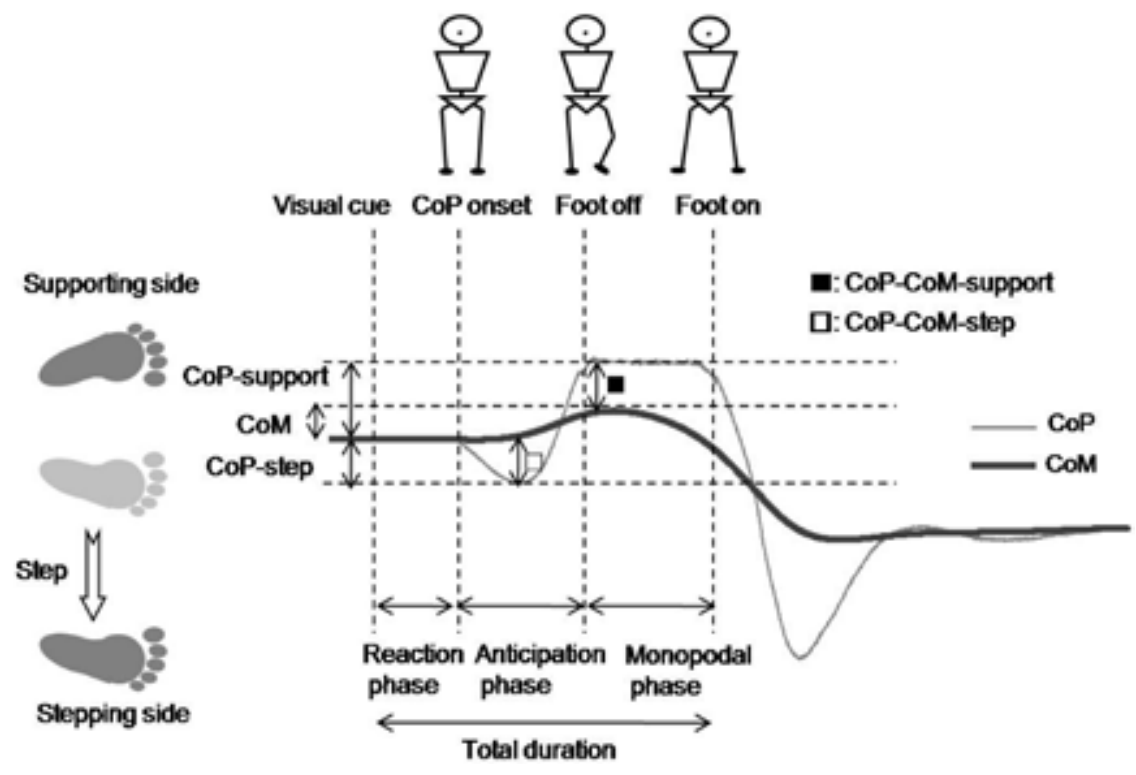

Figure 2 - Mediolateral center of pressure $(\mathrm{CoP})$ and center of mass $(\mathrm{CoM})$ displacements during lateral step motion. The figure also shows temporal and kinetic variables. 
the acceleration trace. The force platform methods that we applied can be considered to provide accurate results for CoM excursion as well as the segmental analysis methods (Ranavolo et al., 2008). The peak displacement of the CoM toward the supporting side during the anticipation phase was calculated from the initial CoP position (Figure 2). In addition, the distances between $\mathrm{CoP}$ and $\mathrm{CoM}$ in the mediolateral direction were calculated at the instant of CoP-step and CoP-support (foot-off), respectively, CoP-CoM-step and CoP-CoM-support (Figure 2). The displacements of $\mathrm{CoP}$ and $\mathrm{CoM}$ were standardized by the transverse diameter of the individual base of support in the initial position.

Four movement durations defined on the basis of kinetic parameters were examined (Figure 2) as follows. (1) Reaction phase: the period from the cue to the onset of the CoP shift in the frontal plane; (2) Anticipation phase: the period from the onset of the CoP shift to the foot-off of the stepping foot; (3) Monopodal phase: the period from the foot-off to the foot-on of the stepping foot; (4) Total duration: the period from the cue to the foot-on.

Because the movements were performed in the frontal plane, only this plane was considered in the kinematic analysis. The peak displacements of the markers placed on the acromion, greater trochanter, and the difference between acromion and greater trochanter on the supporting side were measured during the anticipation phase. The displacements were standardized by the subject's height. The following two angles in the frontal plane were computed: (1) the shoulder angle between the shoulder line (joining the bilateral acromion markers) and the horizontal plane, and (2) the pelvic angle between the pelvic line (joining the bilateral anteriorsuperior iliac spine markers) and the horizontal plane. The variations in the two angles during the anticipation phase were calculated from the initial position. For the displacement and angle variables, displacement and inclination toward the supporting side were expressed as a plus.

To analyze the duration, kinetic, and kinematics data, the mean value of five trials was determined for each subject. The data were expressed as mean $\pm S D$. Student's $t$ test was used to compare the OA group and the control group for all the variables. In both the groups, the relationships between the duration of anticipation phase and the kinetic and kinematic factors which are considered to have a plausible relationship to duration of anticipation phase were examined using Pearson correlation coefficients $(r)$. Statistical significance was accepted at $p<.05$. All statistical procedures were performed using Statistica software (StatSoft, Inc., Tulsa, USA).

\section{Results}

Comparison of temporal variables between both groups is shown in Table 1 . The anticipation phase was significantly longer in the OA group than in the control group. No difference, however, was found in the reaction phase, monopodal phase, and total duration between both the groups.

Comparison of kinetic variables between both the groups is shown in Table 2. During the anticipation phase, both the Fx-support and Fx-step were

Table 1 Temporal variables of the lateral step motion

\begin{tabular}{lccc}
\hline & OA Group & Control Group & P-Value \\
\hline Reaction phase $(\mathrm{ms})$ & $265.7 \pm 44.5$ & $241.0 \pm 51.0$ & 0.19 \\
Anticipation phase $(\mathrm{ms})$ & $418.8 \pm 102.2$ & $347.3 \pm 51.2$ & $0.04^{*}$ \\
Monopodal phase $(\mathrm{ms})$ & $346.2 \pm 127.5$ & $396.9 \pm 87.1$ & 0.28 \\
Total duration $(\mathrm{ms})$ & $1030.8 \pm 213.7$ & $985.2 \pm 117.8$ & 0.54 \\
\hline
\end{tabular}

Note. Values are expressed as mean $\pm S D$. ${ }^{*} p<.05$.

Table 2 Kinetic variables in the anticipatory postural adjustments phase during lateral step motion

\begin{tabular}{lccc}
\hline & OA Group & Control Group & P-Value \\
\hline${\text { Fx-support }(\%)^{\mathrm{a}}}^{\mathrm{a}}$ & $3.5 \pm 1.6$ & $5.1 \pm 2.1$ & $0.03^{*}$ \\
Fx-step $(\%)^{\mathrm{a}}$ & $5.8 \pm 1.2$ & $7.4 \pm 2.1$ & $0.01^{*}$ \\
CoP-step $(\%)^{\mathrm{b}}$ & $12.8 \pm 3.6$ & $14.8 \pm 4.8$ & 0.23 \\
CoP-support $(\%)^{\mathrm{b}}$ & $27.6 \pm 5.5$ & $23.6 \pm 2.6$ & $0.04^{*}$ \\
CoM $(\%)^{\mathrm{b}}$ & $5.4 \pm 4.9$ & $4.9 \pm 3.1$ & 0.81 \\
CoP-CoM-step $(\%)^{\mathrm{b}}$ & $13.5 \pm 4.0$ & $15.8 \pm 4.8$ & 0.17 \\
CoP-CoM-support $(\%)^{\mathrm{b}}$ & $22.3 \pm 6.3$ & $18.6 \pm 3.8$ & 0.11 \\
\hline
\end{tabular}

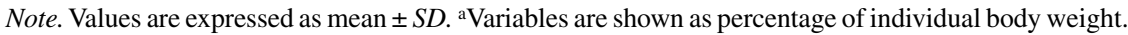

bVariables are shown as percentage of the transverse diameter of the individual base of support. $* p<.05$. 
significantly lesser in the OA group than in the control group. Although the mean values of the initial position of the $\mathrm{CoP}$ before the starting cue slightly shifted to the stepping side in the OA group $(1.1 \pm 5.1 \%)$ as was also found in the control group $(2.1 \pm 2.8 \%)$, there was no significant difference between the two groups. The OA and control groups showed a common pattern in the CoP and $\mathrm{CoM}$ trajectories (Figure 3). Although there was no difference in the displacement of the CoM toward the supporting side between both groups, the CoP-support was significantly greater in the OA group than in the control group. No difference was found in the CoP-step between the groups. The CoP-CoM-step and CoP-CoMsupport showed no significant differences between both the groups.

Although the movement of greater trochanter was not different among groups, the acromion began to move more toward the supporting side in the OA group than in the control group in the anticipation phase. Furthermore, the OA group showed greater difference between the acromion and the greater trochanter than the control group. No difference was observed in the shoulder and pelvic angles (Figure 4).

The results of the correlation analysis between the duration of the anticipation phase and the kinetic and kinematic factors are shown in Table 3. The displacement of the CoM toward the supporting side was positively correlated with the duration of the anticipation phase for both groups. In addition, CoP-CoM-step was also positively correlated with the duration of the anticipation phase in the control group. In the control group, displacement of the acromion and greater trochanter was positively correlated with the duration of the anticipation phase; however, there were no correlations between kinematic variables and duration of anticipation phase in the OA group.
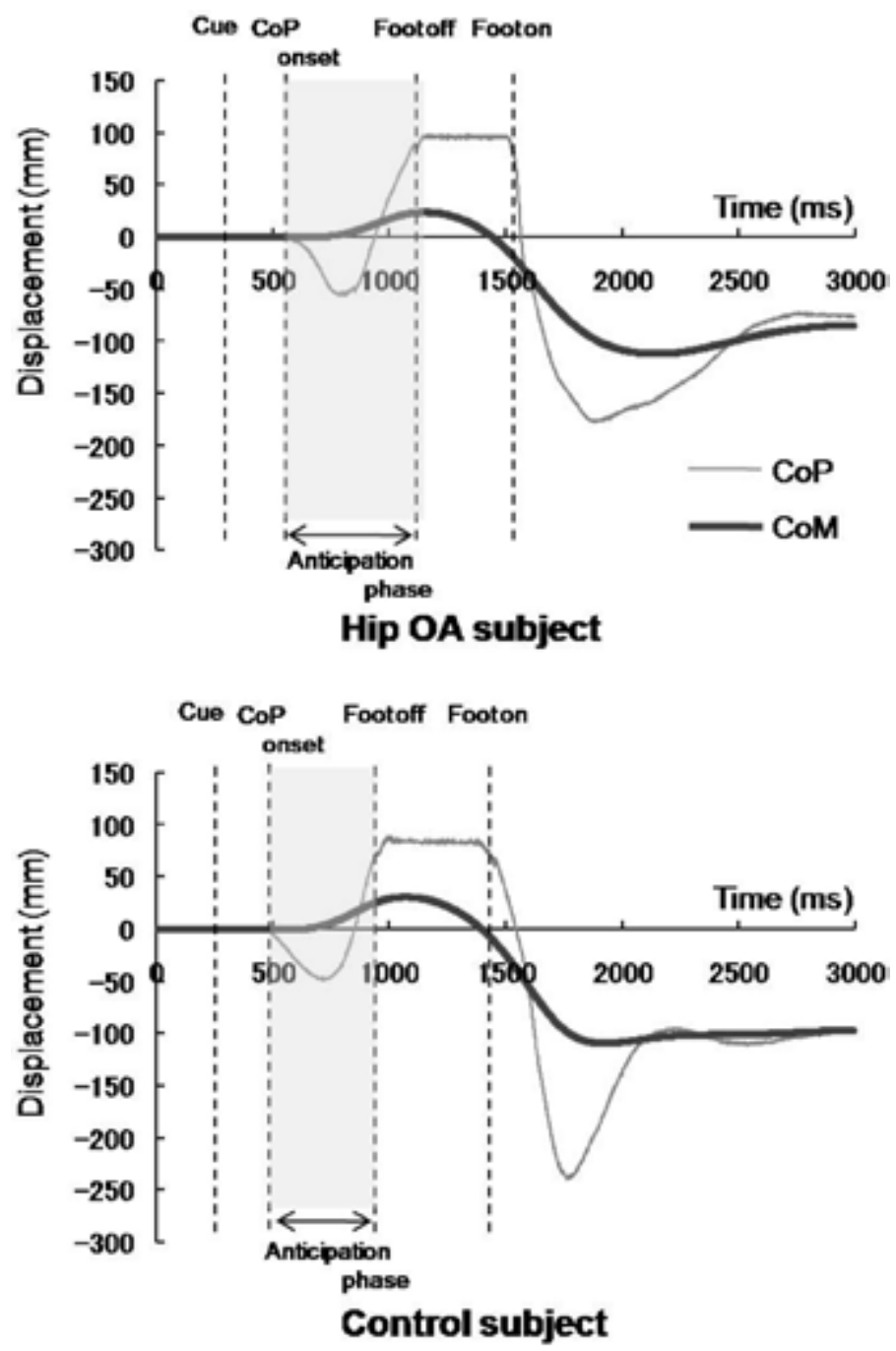

Figure 3 - Typical pattern of trajectories of the $\mathrm{CoP}$ and $\mathrm{CoM}$ in hip OA subject and control subject. The anticipation phase was significantly longer in the OA group than in the control group. 


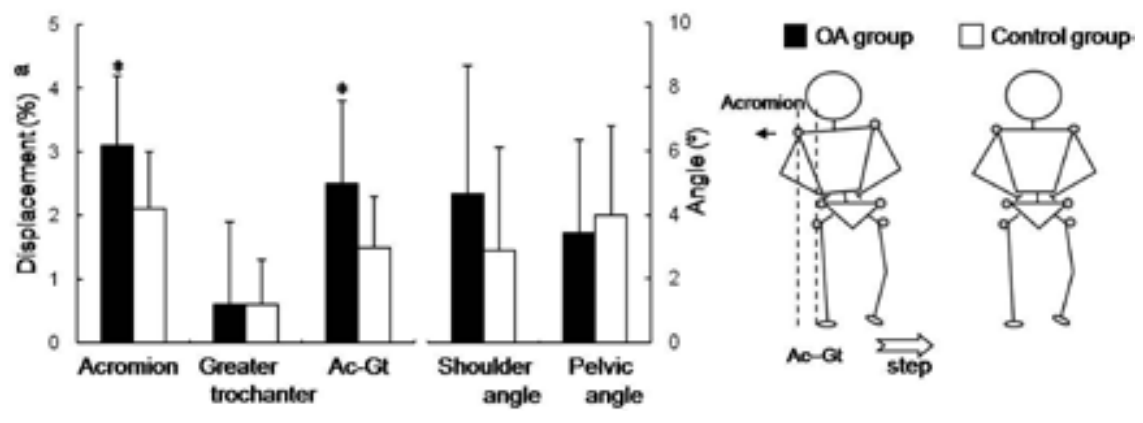

Figure 4 - Kinematic variables in the anticipation phase during lateral step motion. a: variables are shown as percentage of individual height. Ac-Gt: difference between acromion and greater trochanter. $* p<.05$.

Table 3 Pearson's correlation coefficients between the duration of the anticipation phase and the kinetic and kinematic variables in the $O A$ group and control group

\begin{tabular}{lcc}
\hline & OA Group & Control Group \\
\hline Fx-support & 0.28 & 0.44 \\
CoP-step & 0.39 & 0.55 \\
CoM & $0.79^{* *}$ & $0.82^{* *}$ \\
CoP-CoM-step & 0.44 & $0.65^{*}$ \\
Ac displacement & 0.31 & $0.69^{*}$ \\
Gt displacement & 0.43 & $0.78^{* *}$ \\
Ac-Gt difference & -0.18 & 0.12 \\
Shoulder angle & -0.22 & -0.16 \\
Pelvic angle & -0.20 & 0.16 \\
\hline
\end{tabular}

$* p<.05, * * p<.01$.

\section{Discussion}

This study was conducted to identify the movement strategy during the initiation of the lateral step motion in patients with hip OA. Patients with hip OA required a longer duration of anticipation phase than the control subjects before initiating a rapid lateral step. Lateral step is one of the most important postural controls for preventing falls (Maki et al., 1994, Maki \& Mcllroy, 1997, Rogers \& Mille, 2003). Prolonged anticipation phase should be considered an indication of severity in mediolateral dynamic balance control in patients with hip OA.

We instructed the patients to distribute their weight on both legs as evenly as possible, and we believe that the patients were able to distribute their weight almost evenly in the initial standing. Although the commonly tendency was to shift their weight to the sound side, patients in this study were able to stand with their weight almost evenly distributed because most of them had only slight pain in the hip (VAS: $17.1 \pm 17.8 \mathrm{~mm}$ ). It enabled us to compare the motion pattern during the anticipatory phase between the 2 groups.
When comparing the hip OA patients to the control subjects, only the anticipation phase was found to be longer in patients with hip OA. This may be attributed to the pain and stiffness in initiating various voluntary movements reported in patients with hip OA (Bellamy et al., 1999; Bijlsma \& Knahr, 2007; Helve, 2005). Viton et al. (2002) also demonstrated prolonged anticipation phase before initiation of the subsequent lateral step in patients with knee OA. These findings suggest that long anticipation phase is the general strategy for protecting the affected limb and clinicians should focus on the duration of the anticipation phase for assessing the mechanism underlying mediolateral postural control in OA patients.

Along with the longer anticipation phase, the mediolateral ground reaction force which moves the $\mathrm{CoM}$ to the supporting side (Fx-support) and stepping side (Fx-step) was smaller in the hip OA group than in the control group. Meanwhile, the amplitude of the CoM displacement to the supporting side was not different between the two groups. These findings (i.e., longer anticipation phase, smaller force, and same CoM displacement) suggest that subjects with hip OA performed a slower anticipation phase than the control subjects. Shifting of the CoM to the supporting side 
with considerable acceleration in the anticipation phase is accompanied with rapid loading of the supporting leg (the affected leg) which would induce great stress on the affected hip joint. Conversely, if the shift of the CoM toward the supporting side was not sufficient, a large ground reaction force to the stepping direction and a large hip joint torque would be produced. Therefore, both small and large shifts of the CoM to the supporting side would be harmful to the affected hip for patients with hip OA. Our patients with hip OA experienced mild pain on the supporting (affected) hip joint; therefore, they might have adjusted their movement to conditions of the affected hip joint by slowly moving the CoM, which is less stressful on their affected hip joint. Furthermore, the large shift of the CoM to the supporting side induces prolonged anticipation phase since a significant positive correlation was observed for both groups in this study. Thus, if the shift of the CoM to the supporting side was large in the hip OA group, the duration of the anticipation phase might be more prolonged. This slow CoM motion without an increase or decrease in the displacement of the CoM compared with the control subjects probably indicates that patients with hip OA performed slow anticipation phase to achieve both protection of the affected hip joint and quickness in the subsequent lateral step.

Surprisingly, the total duration of the lateral stepping was not different between the two groups even though the anticipation phase was longer in patients with hip OA. In our study, subjects from both groups were required to take steps covering the same distance; thus, it can be considered that CoM displacement toward the stepping side was also the same in both groups. In patients with hip $\mathrm{OA}$, less ground reaction force (Fx-support) might cause a reduction in the acceleration of the CoM to the affected side. This may contribute to the effortless acceleration of the CoM to the stepping side despite the decrease in the force produced by the affected hip joint. For patients with knee OA, Viton et al. (2002) reported that mediolateral ground reaction force that shifts the CoM to the supporting side was increased as compared with the control subjects. Hence, these findings suggest that patients with hip OA who might have decreased mediolateral postural control compared with patients with knee OA achieved rapid stepping by moderating the force generated by the sound limb while protecting the affected hip joint. This coordination between the affected and sound limb may be potentially important to accomplish the protective lateral stepping that is required for standing stability and for avoiding falls in the everyday environment (Rogers \& Mille, 2003).

A characteristic feature of the anticipation phase of the patients with hip OA was found in their trunk kinematics. Although displacement of the CoM toward the supporting side showed no significant differences between the two groups, hip OA patients shifted the acromion to the supporting side with greater magnitude than did the control group, while displacement of the greater trochanter to the supporting side was not significantly different between the two groups. Consequently, the distance between the acromion and greater trochanter was also larger in hip OA patients. Similar phenomena wherein patients with hip OA shift the trunk toward the affected side have been reported as one of the characteristic postures of patients with hip OA (Murray et al., 1971; Thurston, 1985). The force acting on the hip joint is determined by the external moment due to body weight and the counteracting moment of the hip abductor muscles (Dostal et al., 1986). Shifting the trunk to the affected side decreases the moment arm of the gravity of the trunk segment associated with the affected hip joint (Schröter et al., 1999). Our patients with hip OA might have chosen the strategy of shifting their trunk to protect their affected hip joint from a large angular moment associated with the gravity of the trunk. It is likely that this strategy is used to lower the force acting on the affected hip joint as anticipatory postural adjustments. In addition, the displacement of the acromion and greater trochanter was significantly correlated with the duration of the anticipation phase for the control group; however, there was no correlation between movement and duration of the anticipation phase for the patients with hip OA. An interpersonal variability in the trunk motion to protect the affected limb in patients with hip OA might result in the absence of significant correlation between the kinematic variables and duration of the anticipation phase. In contrast, the control subjects might not need a compensatory trunk motion to protect the supporting hip joint. Therefore, they performed the anticipatory postural adjustment while keeping the alignment of the trunk and pelvis, which explained the correlation between the displacement of the acromion and the greater trochanter and the duration of the anticipation phase in the control subjects.

In this study, a detailed analysis such as inverse dynamics to quantify hip torque was not performed. Investigation of the hip torque may provide additional insight into the mechanical stress on the hip joint during anticipation phase and the subsequent lateral stepping movement itself. In addition, we investigated only frontal plane kinetics and kinematics. Since the hip joint is a 3D rotating joint, the anteroposterior component could be altered in patients with hip OA even in the lateral stepping task. Burleigh \& Horak (1996) showed that the duration of the anticipation phase for perturbation-induced step initiation was decreased compared with the stepping in which the cue alone was presented. The duration and movement strategy in the phase of anticipatory postural adjustment in the current study might have been different if the subjects were required to exhibit a step response following external perturbation. Future studies should address these issues.

In conclusion, patients with hip OA performed slow anticipation phase without changing the displacement of the CoM. They showed less mediolateral ground reaction force even in the sound side. Further, our study revealed the greater shift of the trunk during anticipation phase as a characteristic of patients with hip OA. These movement characteristics during anticipation phase might contribute to gaining both protection of the affected hip joint and quickness in the subsequent lateral step. Since disability for mediolateral postural control could be related to falling, therapeutic approaches aimed at allowing patients to step rapidly without experiencing pain should be directed toward adjustment of the CoM motion and trunk motion in an individual-specific manner. 


\section{Acknowledgments}

This study was supported by the Department of Rehabilitation, Osaka Medical College Hospital. The authors are grateful for the contribution of Takahiro Tanaka in data collection. We would also like to thank Masao Furuta for his invaluable technical assistance.

\section{References}

Arnold, C.M., \& Faulkner, R.A. (2007). The history of falls and the association of the timed up and go test to falls and near-falls in older adults with hip osteoarthritis. $B M C$ Geriatrics, 7, 17.

Bellamy, N., Klestov, A., Muirden, K., Kuhnert, P., Do, K.A., O'Gorman, L., et al. (1999). Perceptual variation in categorizing individuals according to American College of Rheumatology classification criteria for hand, knee, and hip osteoarthritis (OA): observations based on an Australian Twin Registry study of OA. The Journal of Rheumatology, 26, 2654-2658.

Bijlsma, J.W.J., \& Knahr, K. (2007). Strategies for the prevention and management of osteoarthritis of the hip and knee. Best Practice \& Research. Clinical Rheumatology, 21, 59-76.

Brenière, Y., Do, M.C., \& Bouisset, S. (1987). Are dynamic phenomena prior to stepping essential to walking? Journal of Motor Behavior, 19, 62-76.

Brown, C.R. (2006). Arthritis and allied conditions. In J.J. Chllaghan, A.G. Rosenberg, \& H.E. Rubash (Eds.), The adult hip (2nd ed., pp. 576-577). New York: Lippincott Williams \& Wilkins.

Burleigh, A., \& Horak, F. (1996). Influence of instruction, prediction, and afferent sensory information on the postural organization of step initiation. Journal of Neurophysiology, $75,1619-1628$.

Dagenais, S., Garbedian, S., \& Wai, E.K. (2009). Systematic review of the prevalence of radiographic primary hip osteoarthritis. Clinical Orthopaedics and Related Research, 467, 623-637.

Dostal, W.F., Soderberg, G.L., \& Andrews, J.G. (1986). Actions of hip muscles. Physical Therapy, 66, 351-361.

Gélat, T., Pellec, A.L., \& Brenière, Y. (2006). Evidence for a common process in gait initiation and stepping on to a new level to reach gait velocity. Experimental Brain Research, 170, 336-344.

Hardcastle, P., \& Nade, S. (1985). The significance of the Trendelenburg test. The Journal of Bone and Joint Surgery. British Volume, 67, 741-746.

Helve, T. (2005). Disease-specific symptoms and signs in patients with inflammatory joint disease. In I. Kunnamo (Ed.), Evidence-based medicine guidelines (pp. 665). San Francisco: Wiley.

Lepers, R., \& Breniére, Y. (1995). The role of anticipatory postural adjustments and gravity in gait initiation. Experimental Brain Research, 107, 118-124.

Liu, W., Kim, S.H., Long, J.T., Pohl, P.S., \& Duncan, P.W. (2003). Anticipatory postural adjustments and the latency of compensatory stepping reactions in humans. Neuroscience Letters, 3, 1-4.

Lyon, I.N., \& Day, B.L. (1997). Control of frontal plane body motion in human stepping. Experimental Brain Research, $115,345-356$.
Maki, B.E., Holliday, P.J., \& Topper, A.K. (1994). A prospective study of postural balance and risk of falling in an ambulatory and independent elderly population. Journal of Gerontology, 49, 72-84.

Maki, B.E., \& McIlroy, W.E. (1997). The role of limb movements in maintaining upright stance: the "change-insupport" strategy. Physical Therapy, 77, 488-507.

Mercer, V.S., Gross, M.T., Sharma, S., \& Weeks, E. (2009). Comparison of gluteus medius muscle electromyographic activity during forward and lateral step-up exercises in older adults. Physical Therapy, 89, 1205-1214.

Murray, M.P., Gore, D.R., Clarkson, B.H., \& Wisconsin, M. (1971). Walking patterns of patients with unilateral hip pain due to osteo-arthritis and avascular necrosis. The Journal of Bone and Joint Surgery. American Volume, 53, 259-274.

Nagasawa, F., Miyane, Y., Akazawa, H., Konishi, Y., Uchida, K., Mitani, S., et al. (2000). Predictability of the progress of secondary osteoarthritis after developmental dislocation of the hip, utilizing inferior edge (of the teardrop) - center (of the femoral head) distance. Journal of Orthopaedic Science, 5, 10-17.

Quintana, J.M., Arostegui, I., Escobar, A., Azkarate, J., Goenaga, I., \& Lafuente, I. (2008). Prevalence of knee and hip osteoarthritis and the appropriateness of joint replacement in an older population. Archives of Internal Medicine, 168, 1576-1584.

Ranavolo, A., Don, R., Cacchio, A., Serrao, M., Paoloni, M., Mangone, M., et al. (2008). Comparison between kinematic and kinetic methods for computing the vertical displacement of the center of mass during human hopping at different frequencies. Journal of Applied Biomechanics, 24, 271-279.

Rogers, M.W., \& Mille, M.L. (2003). Lateral stability and falls in older people. Exercise and Sport Sciences Reviews, 31, 182-187.

Rogers, M.W., \& Pai, Y.C. (1990). Dynamic transitions in stance support accompanying leg flexion movements in man. Experimental Brain Research, 81, 398-402.

Rogers, M.W., \& Pai, Y.C. (1993). Patterns of muscle activation accompanying transitions in stance during rapid leg flexion. Journal of Electromyography and Kinesiology, 3, 149-156.

Schröter, J., Güth, V., Overbeck, M., Rosenbaum, D., \& Winkelmann, W. (1999). The 'Entlastungsgang'. A hip unloading gait as a new conservative therapy for hip pain in the adult. Gait \& Posture, 9, 151-157.

Sharp, I.K., \& England, H. (1961). Acetabular dysplasia. The Journal of Bone and Joint Surgery. British Volume, 43, 268-272.

Shumway-Cook, A., \& Woollacott, M.H. (2001). Normal postural control. In M. Biblis (Ed.), Motor control: theory and practical applications (2nd ed., pp. 163-191). New York: Lippincott Williams \& Wilkins.

Thurston, A.J. (1985). Spinal and pelvic kinematics in osteoarthrosis of the hip joint. Spine, 10, 467-471.

Viton, J.M., Atlani, L., Mesure, S., Massion, J., Franceschi, J.P., Delarque, A., et al. (2002). Reorganization of equilibrium and movement control strategies after total knee arthroplasty. Journal of Rehabilitation Medicine, 34, 12-19.

Watelain, E., Dujardin, F., Babier, F., Dubois, D., \& Allard, P. (2001). Pelvic and lower limb compensatory actions of subjects in an early stage of hip osteoarthritis. Archives of Physical Medicine and Rehabilitation, 82, 1705-1711. 